\title{
System Requirements for an Advanced Cockpit to Reduce Workload and Stress
}

\author{
Paul M. Liston* and Nick McDonald \\ Centre for Innovative Human Systems, School of Psychology, \\ Trinity College, University of Dublin, Ireland \\ \{pliston, nmcdonld\} atcd. ie
}

\begin{abstract}
This paper describes the requirements elicitation process and the subsequent system requirements for an advanced cockpit to reduce crew workload and stress. The paper outlines the need for a step-change in technology and operational practices to ensure the continued safety of a transport system which is predicted to grow. The ACROSS project aims to develop advanced cockpit solutions to reduce workload and stress in an increasingly congested aviation transport system. Six types of requirements were derived including aviate requirements, and navigate, communicate, manage systems, crew monitoring, and crew incapacitation requirements. The research project is currently specifying the human factors requirements for the technologies to achieve improved operational safety.
\end{abstract}

Keywords: requirements, flight deck, flight crew, cockpit, workload, stress.

\section{$1 \quad$ Introduction}

Aviation has achieved an enviable reputation as the leading transport sector in terms of safety. Decades of research and operational innovation have contributed to successive reductions in accident rates. Though there is a decreasing trend in the rate of fatal accidents in the period from 2002 to 2011 to 0.6 per million flights flown (CAA, 2013) the forecasted growth in air traffic of $4.7 \%$ annually (Airbus, 2013) means that aviation accidents will continue to occur on a regular basis.

$52 \%$ of the fatal accidents from 2002 to 2011 involved a flight-crew related primary causal factor. Indeed, seven of the top-ten causal factors of all fatal accidents in this period came from the flight crew. The most frequently allocated causal factors were "Flight-handling" and "Omission of action or inappropriate action" (CAA, 2013).

\subsection{The Challenge}

As technology has progressed aviation accidents based solely on technical faults or problems have decreased. Indeed it is an entirely natural trajectory for accident

\footnotetext{
* "The research leading to these results has received funding from the European Community's Seventh Framework Programme FP7/2007-2013 under grant agreement no. ACP2-GA-2012314501". ACROSS Project Website: www.across-fp7.eu

D. Harris (Ed.): EPCE 2014, LNAI 8532, pp. 34 41, 2014.

(C) Springer International Publishing Switzerland 2014
} 
statistics to increasingly implicate the human factor as technology develops. In the case of flight operations the humans in the cockpit are the factors introducing variability of performance. However, separating out the technology from the human operator - in this case the pilots - may not be useful in helping to understand why accidents and incidents occur. One possible reason for the high rate of flight-crew related accidents could be the increased complexity of aircraft systems. "Flight Crew Perception and Decision-making" (i.e. omission of action and inappropriate action) is the number one causal factor allocated for all fatal accidents for the ten-year period 2002 to 2011 (UK CAA). Research has shown that automation in the cockpit has led to issues relating to safety and situational awareness in particular (Mosier et al., 2013; Dao et al., 2009).

The predicted growth in air transport brings about further challenges. Not only are aircraft becoming more complicated, but the airspace and airports are becoming increasingly crowded (something compounded when weather conditions become suboptimal). Couple this with the fact that the flight phases that have a high workload (approach, landing, turn-around and take-off) are happening in, and around, airports and it is clear that pilot stress and peak workload are key safety challenges for the aviation sector.

\subsection{The Need}

A step-change in technology and flight operations practices is needed to meet the aforementioned challenges. This step-change should not supplant ACARE SRA2's goal of increasing safety by a factor of 5 compared to the situation in 2000, but should supplement it and ensure that humans and machines work together effectively in the aviation system. Airframers and equipment suppliers have already focused their attention on reducing the complexity of aircraft technology and some of these solutions to improve cockpit operations have been implemented in new generation aircraft such as the Boeing B787 and the Airbus A380.

Despite these efforts certain combinations of unpredictable situations, such as difficult meteorological conditions, multiple system failures or cockpit crew incapacitation, can lead to peak workload conditions. These situations are difficult to anticipate and the number of actions that flight crew have to simultaneously execute and the amount of information they have to process can quickly render the workload unacceptably high. Given that accidents are more likely to occur when flight-crew workload is high, improving crew performance in peak workload conditions is thus critical to enhance safety. Clearly a more integrated, comprehensive solution is needed to address not just complexity, but also peak workload and stress.

\subsection{The Solution}

The achievement of an overall reduction in the number of aviation accidents necessitates the design and implementation of new solutions (based on both hardware and software) to allow flight crew to more easily manage peak workload situations. To this end, the ACROSS research project aims to make important safety gains by 
developing an advanced cockpit to reduce workload and stress. Ensuring safe operations under crew peak workload can be achieved by providing a cockpit environment that mitigates the risk of human error on the flight deck and by limiting crew workload to ensure that pilots will have the opportunity to address all relevant issues in a timely and appropriate manner - thereby mitigating the risk of pilot error. Flight crew incapacitation is one circumstance in which the remaining pilot has to manage the situation under significant stress. In 2004 pilot incapacitation occurred on 36 occasions (Evans \& Radcliffe, 2012). Pilot incapacitation can come about for various reasons and the ACROSS project intends to develop technologies which will help the remaining crew to manage these unplanned situations of reduced crew and to ensure the safe completion of the flight.

\subsection{ACROSS Objectives}

The ACROSS project has three main objectives which inform all research and development activities. They are:

1. Facilitate the management of peak crew workload situations during a flight

2. Allow reduced crew operations

3. Identify open issues for possible single-pilot operations

\section{ACROSS Objective 1: Crew under peak workload situations}

This objective targets fully capacitated crew with peak workload and will develop and demonstrate solutions up to Technology Readiness Level (TRL) 5 (Component and/or mock-up validation in a relevant environment).

\section{ACROSS Objective 2: Reduced crew operations}

This objective targets three conditions of reduced crew and will develop and demonstrate solutions up to TRL 3 (Analytical and experimental critical functions and/or characteristics proof-of-concept);

1. Long haul flight with reduced crew;

2. One pilot incapacitated;

3. Short/medium range flight with both pilots incapacitated.

\section{ACROSS Objective 3: Open issues for possible single-pilot operation}

Single-pilot operations in all conditions are considered a long-term evolution that is not in the scope of the ACROSS project. The project consortium considers singlepilot operations as a case study that stimulates innovation and facilitates the identification of solutions that could be used to improve the current safety level in situations of peak workload and reduced crew. Any solutions developed to manage peak workload and reduced crew situations may be considered for possible single pilot operations in the future. 


\section{ACROSS Requirements}

The first step in realising the three ACROSS objectives was to elicit requirements for the systems which can help improve crew performance in peak workload and stressful conditions. 12 stakeholder organisations were involved in generating and specifying the requirements (these included aircraft manufacturers, aerospace safety service providers, navigational information providers, communication, crew and fleet management services providers, and cockpit communication services providers). Each stakeholder organisation was responsible for producing requirements according to their area of expertise.

\subsection{ACROSS Requirements Process}

Step 1 - Selecting the Scenario

The ACROSS project identified 29 scenarios based on accidents and current safety threats that were selected as relevant due to their implications for stress, workload and incapacitation on the flight deck. As such these scenarios were the starting point for the requirements elicitation process.

Step 2 - Using the Scenario as a reference

When the requirement authors were writing the requirement they were instructed to keep the specifics of the scenario in mind. The use of scenarios to guide the requirements process creates a constant link from the overall goals of the project right the way through evaluation and validation.

\section{Step 3 - Completing the "Requirements Capture Form"}

A 'Requirements Capture Form' was developed in order to gather the information about not just the system requirement but also the operations, processes and systems implicated. In addition the form structure ensured the requirements were linked to scenarios and objectives. Guidelines on how to write a good requirement (Kar \& Bailey, 1996) were made available to the partners.

Step 4 - Collating and analysing the requirements

All requirements received were reviewed for completeness and clarity.

Step 5 - Requirement Stakeholder Clarification Interview

Teleconference interviews (guided by a standardised interview schedule) were held with the requirement authors. The objective was to refine and/or clarify any ambiguities or errors in the requirement text and to understand completely the background to the requirement - ensuring all sections of the 'Requirement Capture Form' were completed. Additional information about processes affected by the requirement, together with predicted impact were elicited. 
Step 6 - External Experts Review

External experts (especially representing flight operations and ATM (air traffic management)) were presented with a selection of the collated requirements in order to solicit feedback from stakeholder perspectives not present in the requirement author group. The objective was to get feedback and suggestions for improvement from experts external to the project (and in particular those with operational experience).

Step 7 - Gap Analysis

A gap analysis was performed - the objective of which was to ensure that all crucial areas of interest had related requirements. All project stakeholders participated in the gap analysis - reviewing both the content of individual requirements and the overall scope of the requirements in their entirety. Requirement authors had the opportunity to accept or reject (citing reasons) any comments or suggestions. This process was managed using a Gap Analysis Protocol form and another one for Gap Analysis Resolution.

Step 8 - Fill Gaps Identified and Review

This was the last step in the requirements capture process. Any suggestions which were accepted at the Gap Analysis stage were actioned and reviewed before the requirements could be considered final.

\section{Results - The Requirements}

139 Requirement Capture Forms were elicited in Step 3 of the process. At the end of the gap analysis process - following the combination of requirements due to repetition and the specification of new requirements - there were 123 final requirements (see Table 1 for their distribution across the project's technical functions).

Table 1. Final ACROSS Requirements by Technical Function

\section{Technical Function Number of Final Requirements}

Aviate

Navigate

Communicate

Manage Systems

Crew monitoring

10

Crew incapacitation 
A selection of the final requirements are listed below (italicised).

\section{Aviate}

Aviate requirements relate to the task of flying the aircraft according to operational requirements, supported by monitoring from the pilot not flying.

- $\quad$ The system shall assist the crew in performing manual tasks during the execution of initial climb.

- The system shall assist the crew when having to perform an emergency descent towards nearest, suitable airport in difficult terrain.

\section{Navigate}

Navigate requirements relate to monitoring threats to the flight plan from weather, traffic, loss of infrastructure capability, and adapting the flight plan if necessary.

- The system shall provide more functional and less physical references regarding aircraft status (mask the complexity of the system)

- The system shall offer the capability for the ground to support the remaining pilot to handle the situation.

\section{Communicate}

Communicate requirements relate to maintaining contact between the crew and ATC (Air Traffic Control), cabin staff (if applicable), and the AOC (Airline Operations Centre).

- The system shall maintain air-ground communication without requiring actions by the cockpit crew or ground crew.

- $\quad$ The system shall be robust against intentional interference (i.e. jamming) and non-intentional interference.

\section{Manage Systems}

Manage systems requirements relate to monitoring, evaluating (and reconfiguring if necessary) the aircraft's systems status to ensure optimum efficiency and safety.

- The cockpit and all its systems shall provide the relevant information through a functional view regarding aircraft remaining resources/performance, especially in case of abnormal or unexpected events.

- The system shall prioritize and filter EICAS / ECAM messages (Engine Indication and Crew Alerting System / Electronic Centralized Aircraft Monitor).

\section{Crew-monitoring}

Crew-monitoring requirements relate to providing crew monitoring functions for the evaluation of the crew's physiological and behavioural condition as they operate the systems, and to adequately address peak workload situations and reduced crew operations. 
- The system shall be able to detect clues of vigilance loss, fatigue or peak workload.

- The system shall make the crew aware of their physiological and behavioural condition without being physically or psychologically intrusive.

\section{Crew-incapacitation}

Crew-monitoring requirements relate to the extreme situation of incapacitated crew, which is encountered very rarely in a two-pilot configuration, and involve identifying and developing automatic functions and safety nets.

- The system shall be capable of ensuring the continued safe flight and landing of the aircraft without any flight crew intervention after the start of take-off in normal conditions.

- The system shall automatically detect when one or more of the flight crew are incapacitated.

\section{Design and Development Implications}

Eliciting requirements from scenarios is a recognised practice in requirements engineering. The approach outlined in this paper supplemented this practice with early consultation with a wide group of end-users beyond just the technology development team and the stakeholders represented in the project consortium.

Looking at the number of requirements elicited per technical function one could make an inference about the relative complexity of one proposed technical solution over another. Indeed 'Crew Incapacitation' requirements number 49, while the next function in order of number of requirements elicited is 'Navigate', with 27 requirements. Clearly the technology needed to resolve a situation where both pilots are incapacitated represents a step-change and is certainly cutting-edge but it doesn't mean that it is necessarily a more demanding proposition than supporting the navigation of an aircraft in peak workload conditions just because of the number of requirements elicited. At the outset of the requirements elicitation process the stakeholders were encouraged to define what was meant by "the system" in each requirement. In the clarification interviews it became clear that some partners were choosing to not define "the system", not to introduce ambiguity, but to build in a level of flexibility. In so doing no particular solution is precluded a priori. Given that a single requirement can impact more than one project objective or technical function it was considered prudent to have this level of flexibility at the requirements stage. Later, once the development work is underway the requirements can be specified in terms of what is meant, in that context, by "the system". Some of the requirements which were derived are at a 'high level' and others are more detailed and technical. At this initial stage this is not necessarily a limitation as in the 'Requirement Capture Form' all details relating to the requirement are captured - especially details about which objective is linked to each requirement. In this way those requirements that are overarching (and as such relate to all objectives) will feature in all targeted solutions. Those requirements which are very detailed and specific will only be passed on to those solutions that are related to them. 
The next step for this project involves the specification of human factors requirements related to the system requirements detailed herein - how do the systems and processes that constitute the aviation transport system as it currently exists need to change in order to support the new technologies and solutions that will be developed in the ACROSS project? Parts of the information required to answer this question were elicited as part of the clarification interview (specifically information related to operational practices and processes) and the next step in the research involves gathering this information in a more systematic way so that the technology development work can be informed by a human factors-led agenda.

\section{References}

1. Airbus, Global Market Forecast (2013), http: / / www . airbus. com/company/market/forecast/

2. CAA. CAP 1036 - Global Fatal Accident Review 2002 to 2011, UK CAA (2013)

3. Dao, A.-Q.V., Brandt, S.L., Battiste, V., Vu, K.-P.L., Strybel, T., Johnson, W.W.: The Impact of Automation Assisted Aircraft Separation on Situation Awareness. In: Salvendy, G., Smith, M.J. (eds.) Human Interface, Part II, HCII 2009. LNCS, vol. 5618, pp. 738-747. Springer, Heidelberg (2009), http: / / human-factors . arc .nasa.gov/ publications/Dao_et_al_ImpactofAuto_HCII09.pdf

4. Evans, S., Radcliffe, S.A.: The annual incapacitation rate of commercial pilots. Aviat. Space Environ. Med. 83, 42-49 (2012)

5. Kar, P., Bailey, M.: Characteristics of Good Requirements. International Council of Systems Engineers, Requirements Working Group. INCOSE Symposium (1996)

6. Mosier, K.L., Ute Fischer, U., Morrow, D., Feigh, K.M., Durso, F.T., Sullivan, K., Pop, V.: Automation, Task and Context Features Impacts on Pilots' Judgments on HumanAutomation Interaction. Journal of Cognitive Engineering and Decision Making 7, 377-399 (2013), doi:10.1177/1555343413487178 